\title{
INFORME SOBRE LA ESCUELA NACIONAL UNIFICADA ${ }^{1}$
}

\section{Ministerio de Educación Pública - Superintendencia}

La Escuela Nacional Unificada (ENU) fue un proyecto de transformación de la educación chilena impulsado por el gobierno de la Unidad Popular. El carácter de este proyecto era integral pues concebía que la educación era una necesidad vital, es decir, que debía desarrollarse permanentemente desde el nacimiento a la vejez. El extracto del informe que aquí se presenta está firmado por el profesor de Estado en Historia, Geografia y Educación Cívica egresado del Instituto Pedagógico y, posteriormente, académico de la Universidad de Chile, Iván Núñez Prieto (1932), Premio Nacional de Educación 2015, nombrado Superintendente de Educación en el año 1970. En el extremo superior derecho de este manuscrito mecanografiado, puede leerse: "Último informe. 23 de febrero de 1973".

Es un documento institucional de la Superintendencia del Ministerio de Educación Pública, que establecía los lineamientos centrales que tendría la nueva política educativa que apuntaba a la puesta en práctica de un modelo de desarrollo cultural revolucionario, el que debía contribuir a la construcción de "una sociedad socialista, humanista, basada en el desenvolvimiento de las fuerzas productivas, en la superación de la dependencia económica, tecnológica y cultural; en el establecimiento de nuevas relaciones de propiedad; y en una auténtica democracia y justicia social garantizadas por el ejercicio efectivo del poder del pueblo".

El objetivo de la ENU era transformar la educación capitalista "tradicional, autoritaria, competitiva, clasista e individualista, centrada en generar individuos descomprometidos, egoístas y centrados en su propio éxito”. El ideal era crear un sistema único de educación: único porque subrayaba la unidad y armonía que debe tener el proceso de crecimiento psicosocial y biológico de cada una de las personas; nacional porque nacía del esfuerzo intelectual, social y material de los chilenos; diversificado pues debía adaptarse a las condiciones de cada región; democrático porque correspondía basarse en la participación ciudadana, pluralista y no doctrinaria.

1. Ministerio de Educación Pública, Superintendencia (1973). Informe sobre Escuela Nacional Unificada. Biblioteca digital del MINEDUC. Santiago: Ministerio de Educación, Gobierno de Chile. Disponible en: https://bibliotecadigital.mineduc.cl/handle/20.500.12365/79 
MINISTERIO DE EDUCACION PUBLICA SUE TRINTENDENCIA

I N F O R M E

$\mathrm{S} O \mathrm{i} \mathrm{R} \mathrm{E}$

ESCUEL A NACIONAL UNIFICADA

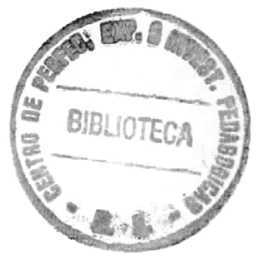

Santiago, Enero de 1973.- 
En conformidad con las disposiciones legales vigentes, el Ministorio do Educación prosenta esto "Informe subre Escuola Nacional Unificadal a consideración del Honorable Consojo Nacional de Educación y, a través de ól, a los divorsos organismos de la comunidad.

Ia voluntad unánime dol Primor Congreso Nacional do Educación, so pronunció sobrc la necesidad do construir la Escuola Nacional Unificada. En dicho tornoo se definioron las líneas centralos do la nueva organización escolar quo Chile requoría. El Ministorio de Educación cumplo con ol mandato de la comunidad y se dispone a iniciar, en 1973, el proceso de desarrollo de la Escuela Nacional Unificada.

Con la entroga de este documento, fruto de estudios técnicos, ordenados por el Comitó Coordinador de los Servicios del Ministerio, so abre un debato que permitirá procisar y enriquecer, con ol valioso aporte do la comunidad, las formas concrotas de llevar a cabo el propósito de instituir la Escuola Unificada.

las nuevas formas educativas surgirán do una masiva combinación de refloxión y praxis de varios años por parto de la comunidad on su conjunto. Particularmente, la Escuela Necional Unificada será el iesultado de un colectivo proceso de búsqueda. de las modalidados óptimas que se extraigan de nuestra rica potenoialidad de canbio.

Con este Informe, queremos motivar y orientar la discusión y olaboración culectiva y roflejar los criterios con que el Ǵobierno encarará. la tarea que le sugiriera el Primer Congreso Nacional de Educación.

Naturalmente, una problemática tan compleja como la transformación radical de la uscuola chilena no puedo agotarse en los escuotos márgenos de este Informo.

La Escuela Nacional Unificada es parte importante de una politica educacional mucho más vasta, cuyas líneas generales actualizadas se encontrarán en el discurso que pronunciará el compañero Jorge Tapia Valdés, Ministro de Educación, con motivo de la entrega oficial del Informe. 
La nueva institución que aquí proponemos se insertará un proyocto quo cubra todas las necesidades educativas del pueblo de Chilo, a través do un verdadero Sistuma Nacional de Educación que haga realidad la educación pormanento. Habrá un documento conplementurio sobro esta materia.

Del mismo modo, habrá documentos complenentarios sobro los fundamontos de la iscuola Unificada, es decir, una síntesis de los antecdentes históricos, nacionales e internacionales y de los rasgos de la crisis de fondo del complejo "suciedad-educación"s sobre el desarrollo curricular, particularmente de los tramos que se reformarán en 1973; sobre los ensayos de nueva orgunización oscolar que so emprenderán de inmediato sobro la participación de la comunidad ys especialmente, de los trabajadores de la educación en los cambios que se proponen, etco

El Ministerio de Educación invita, en consecuencia, a estudiar y debatir con genorosidad $y$ actitud constructiva los mejores caminos para hacer realidad esta transformación proyectada como una de las grandes metas de la nación chilena.

IVAN NUITEZ PRIETO

Superintendente de Educación Pública. 


\section{Fágs.}

1. Un Sistema Nacional pura la Educación

Permanente en una Sociedad de

Transición al Socialismo ..................... 1

2. Fundamentos $\ldots \ldots \ldots \ldots \ldots \ldots \ldots \ldots \ldots \ldots \ldots \ldots \ldots \ldots . \ldots \ldots$

3. Caracterización .............................. 5

4. objetivos ................................ 6

5. Estructura ................................. 8

6. Estructuración Orgínica de la

Escuela Nacional Unificada ................... 12

7. Acciones y Roquisitos para la

Puesta on Marcha de la

Escuela Nacional Unificada 
1. UN SISTPGMA NACIONAL PARA LA EDUCACION PRRMANENTE

EN UNA SOCIEDAD DE TRANSICION AL SOCIALISMO.

1.1 La perspoctiva estratégica que ilumina la nueva politica educacional presupone la construcción de una sociedad socialistą humanista, basada en ol desenvolvimiento de las fuerzas productivas, en la suporación de la dependencia econónica, tecnológica y cultural, en el establecimionto de nuevas relaciones de propiodad y en una auténtica democracia y justicia social garantizadas por el ejercicio efectivo del poder por el pueblo.

1.2. A esta concepción del desarrollo nacional debe correspondor un sistema educacional cuantitativa y cualitativamente diferento del actual con una cobertura que abarque a la población en su conjunto y no solo a las nuevas generaciones. Fón otros términos, un Sistema Nacional que haga realidad la atención oducacional a los individuos desde el nacimiento hasta la ancianidad, ya que on todas las fases del desarro110 individual hay necesidades que pueden y deben ser satisfochas a través de la educación. Esto es "educación permanente".

1.3. Pntendemos también la educación permanonte, como una educación do masas, por las masas y para las masas, on una sociodad como la socialismo, on, que la comunidad va progresivamente organizándose para asumir colectivamente la responsabilidad de oducar a sus miembros, mientras que las instituciones regulares de enseñanza que hasta ahora han mantonido una especie de monopolio educacional inu caro a las minorías dominantes, van paulatinamente suprimiendo las barreras que las separan de la vida social concreta $\theta$ intengrándose realmente a ella.

1.4. La educación permanente constituye, por lo demás, la única respuesta. viablo a los requerimientos de una sociedad on tránsito revolucionario, que forma parto de una Fumanidad que avanza aceleradamente hacia el siglo XXI, on quo tanto ol conocimionto científico, como las tecnologías, las relacionos económicas y las formas sociales se ronuevan con tal rapidez quo toda la educación formal quo pronto en retraso. Fntendemos, on consecuencia, la educación permanente como una habilitación contínua del hombre para crear y participar del cambio social, económico y cultural.

1.5. Con estos objetivos, el Sistema Nacional de Educación ha de apoyarso on $10 s$ más avanzados logros culturales, científicos y tecnológicos para planificarse en armónica integración con el planeamiento globai de la sociedad, do acuerdo a criterios de unidad, continuidad, diversificación y democratización.

1.6. Si bien esta es una tarea estratógica de la nación chilena, a consegrir a plazo mediato, la edificación dol Sistema Nacional de Educación, debe comenzar desdé ya, a partir de los matoriales legados por la oducación tradicional. En consocuencia, doben transformarse los actualos niveles parvulario, básico y medio en el Area de Educación Regular del proyectado Sistema Nacional y, paralelamente, debe conformarse el Area do Educación Extra-escolar del mismo, para cubrir las nocesidades educativas del restante sector no atendido por la onseñanza formal: 
1.6.1. El Area de Educación Regular incluiré como un todo planificado, contínuo y divorsificado, los actuales subsistemas de educación freoscolar, básico y modio, resolviendo a la brevodad las contradicciones de clases hasta hoy impurantos. Esta área tione por finalidad entregar foración general politécnica y profesional a las nuevas genoraciones conforme a los requorimiontos do los divorsos momontos dol desarrollo sioobiológico do los educandos.

1.6.2. El Area do Educación Extra-oscolar so constituirá a partir do la coordinación de las actuales aociones llovedas a cabo por los sorvicios de educación de adultos del Ministorio, las univorsidados, INACAP, Municipalidados, servicios públicos, Contral Unica do Trabajadoros y sindicatos, organizaciones comunitarias, culturales, politicas y roligiosas, con la colaboración do los medios de comunicación de masas y las ompresas productivas. Esta área irá progrosivamente desarrollándose, bajo una planificacion muy flexible, para cubrir on primera prioridad las nocosidados educacionalos do los inoscolares y analfabotos, los desortores de la enseñanza regular, los trabajadores que roquioren una superación cultural y tecnológica consonante con su nuevo rol, los scotoros de irregulares físicos y socialos, las comunidades locales, especialmento las más rotrasadas, otc. Todo ollo sin olvidar las necesidados de recalificación y perfeccionamionto de quiones rocibieron una oducación rogular nás - menos cumpleta.

1.6.3. La división on dos áreas es dictada por las condiciones do partida en al proceso de estructuración del sistema. Tieno, por tanto, caráctor transitorio e iré progresivamente disolviéndoso on la modida en que so desarrollen las condiciones favorables a la edificación del sistema como un todo.

En este mismo instante existon zonas de contacto que relativizan la estuucturación on dos áfoas por ejemplo, los actualos servicios de oducación de adultos dol Ministerio do Educación quo ofrocen enseñenza regular a un sector al que corresponde una oferta del tipo extra-escolar, otro ejemplo, las iniciativas para ontrogar una atención educacional no sistemática a los adolescontes a través do los programas dol Departamento do Educación Extra-cscolar del Ministerio, etc。

1.6.4 Las universidades során centros superioros de investigación, creación y docencia on que culminarán los procesos de educación permanente quo se canalicen por las áreas regular y extra-escolar. Fn consecuoncia, sin desmedro de su función cultural y cientifica de alto nivel, se podrá accedor a ellas desdo la educación rogular y dosde el campo del trabajo, acroditando los requisitos acadómicos necesarios para ol reseuardo de su oxcelencic.

1.7. La Escuela Nacional Unificada se insertará on el área de oducación regular dol Sistoma Nacional y se orientarés específicamonte a ofrocer atención oducativa integral a las nuovas generacionos de niños y adoloscentes, desde su paso por la. Sala Cuna hasta su egreso hacia ol trabajo productivo, hacia los estudios superiores o hacia una combinación o alternancia de ambos.

1.8. Si bien coincide con conceptos formalmonte acoptados a oscala internacional, ol Sistema Nacional de Educación se construirá a partir do la experioncia y de la capacidad de respuesta del pueblo chileno, en ol contexto del proyecto socialista-democrático de desarrollo del país. La Escuola Nacional Unificada es, pues, una iniciativa quo so dosenvolverá dentro de una dinámica condicionante mocho más amplia. 


\section{IUNDAMENTOS}

La Escuela Nacional Unificada se construye para resolver en forma positiva la profunda crisis estructural de la oducación que so viene arrastrando dosde hace largo tiompo y que so exprosa on:

2. 1. La contradicción entre ol creciente procoso de socialización de las relaciones uconómicas, socialos y, políticas (productos de factores tales como la industrialización, la urbanización, la explosión demográfica y la revolución ciontífico-tconologica) y la incapacidad de un sistema oducacional clasista 0 individualista para responder a los requerimiontos educativos que eso proceso conllova y que, por el contrario, siguo promoviendo una ideología capitalista anacrónica.

2.2. La contradicción entro ol cada vez más vigoroso impulso popular a los procosos do participación y solidaridad social y un sistoma educacional autoritario, competitivo y tradicionalista, destinado a producir individuos descomprometidos, egóistas y centrados on su propio śxito.

2.3. La contradicción ontre el ascenso del pueblo a travós de una permanento lucha por mayores conquistas socialus quo signifiquon una incorporación real de los trabajadoros a mejores niveles de vida y un sistoma oducacional discriminatorio $\theta$ injusto que, en los hochos, sigue notando a la mayoría del pueblo el accoso a la cducación o discriminéndolo on la calidad do la que logra recibir.

2.4. La contradiccion ontre la podorosa lucha que libra el pueblo para hacer de chilo una sociadad ofoctivamente domocrática on que ol podor lo ejorzan las erandes mayorías y un sistema educacional disoñado para. reproducir la sociodad de clases y su consiguiente sistema do dominación de las mayorias por las minorías y do explotación dol hombro por ol hombre.

2.5. La contradicción entro las crociontos exigoncias cientificas y técnicas de la lucha contra las raices dol subdesarrollo y el deterioro do la capacidad de evolución ciontifico-técnica de la actual organización oscolar.

2.6. La contradicción entro una sociodad quo aspira a construir su cultura on la valorización del trabajo productivo como fuente do la roalización personal y de la riqueza nacional y una educacion que desprecia al trabaji on favor del consumo como signo de prestigio y dignidad social.

2.7. La contradicción ontre las exigencias do calificación laboral del pueblo como condición básica do un mojoramiento do la productividad dol sistema oconómico y un sistoma educacional quo desvaloriza el trabajo productivo y no otorga, a la immunsa mayoría ninguna capacitación espocial para ejercorlo.

2.8. La contradicción ontre las nocesidades de la organización, la planificación y la administración que implica el desarrollo on una sociedad democrática y ol caráctor contralizado, autoritario y compartimentado dol sistoma administrativo oducacional quo favoreco el dosarollo del 
burocratismo vorticalista, el quo junto con ahogar al potencial popular y la consideración de las nucesidados particulares do cada región, frona la oxpansión do los servicios $y$ su mejoramiento y coarta ol dosarrollo de las potoncialidados resionalos y localos dol sistoma.

2.9. La contradicción ontro las exigencias do un desarrollo acolerado do la ciencia y la têcnica más avanzada para superar la dopendencia ciontífica y tocnológica y la absorción do casi toda le actividad del osfuorzo universitario on la taroa de dar algún destino a la vida do los jóvenes ogresados de un sistema educacional quo no los habilita para hacor frento a la vida del trabajo.

Las contradiccionos anotadas so ven hoy dia acentuadas y multiplicadas, amenazándo con transformarse un ol talón do Aquilos dol procoso do dosarrollo chilono, pues on estos últimos años el pals ha iniciado un profundo proceso de cambios ostructurales cuyo éxito doponde cada vez más de la capacidad que ol pueblo tenga para enfrenter y resolver cada uno de los desafíos plenteados on el presente.

El procoso do domocratización del país ha implicado cambios radicalos on la conomí, lo quo ha dado lugar, a la creación dol ároa social de la economía quo comprendo sectoros industriales, mineros, agricolas, financieros y de servicios.

El árca social constituyo la posibilidad real do la incorporación do los trabajadores al podor y al Gobiorgo de las grandes mayorías, y al mismo tiempo deberá ampliar las oportunidades de trabajo a través do la acoloración dol proceso do dosarrollo.

Esto procoso trao consiguo un doblo compromiso a la Ecucación Chilonas por una parte pone de manifiesto las incapacidados y doficiencias dol sistema educacional para contribuir al éxito do las taroas que ol puoblo hoy so planteay, por otra parte constituye una oportunidad histórica para desarrollar un cambio profundo del sistema oducacional que lo permita superar las contradiccionos quo so anotaron més arriba.

Para esa taroa, la oducación chilena cuonta con los rocursos necosarios y suficiontos:

a) Poseo una valiosa tradición do investigación y cambio dol maçisterio chilono $y$ do los ospecialistas en oducación, que junto con estudiar y luchar con las transformacionos sociales, han ensayado, con divorsa suerte, toda una gnme do innovaciones concrotas.

b) Poseo un vasto conocimiento do la oxporiencia internacional do transformación oducacional, que recomionda polfticas do unificación del sistema educacional y de integración ontre onseñanza y producción similares al intonto que se busca con la Escuola Nacional Unificada.

c) Poseo el inaprociablo apoyo de la comanidad nacional manifostado en las conclusiones unánimos del Conereso Nacional de Educación y en la movilización constanto do los padres de familia, los ostudiantos, los trabajadores en gonoral y la tradición pedagógica y de lucha do los trabajadores de la educación. 


\section{3.- CARACTERIZACION}

La Escuola Nacional Unificada so pondrá on marcha on un procoso de cuatro años y tendrá un carácter permanento de onsayo y bísquoda de las mejoros rospuestas a los cambiantes problemas quo la vida social plantea a la educación.

La Escucla Nacional Unificada sorá:

3.1. Nacional, porque nace del esfuorzo inteloctual, social y matorial de la comunidad chilena y do su historia, y porquo contribuirá al fortalecimiento do nuestra identidad y soberanía.

3.2. Unificada, porque hará suya la unidad del procoso de crecimionto psico-biológico y social del ser humano, supondrá el desarrollo do una cultura fundada on la unidad ontro teoría y práctica, y entre educación y vida $\mathrm{y}_{9}$ on consocuoncia, tondrá un carácter contínuo, superando la compartimontación ontre onseñanza parvularie, básica y mediag intogrará los actuales canalos científico-humanistico y tócnico-profosional; $\mathrm{y}$ so realizara on $\mathrm{y}$ dosdo la comunidad.

3.3. Diversificada, porquo atendorá las nocosidados diforonciadas dol dosarrollo nacional, responderá a los roquerimiontos dosigualos do las regionos y comunidades locales y atendorá a las exigoncias dol desarrollo individual, compatibilizándolos armónicamento.

3.4. Democrática, porquo se basará on la participación du la comunidad on su gostación y dosarrollo, porquo ampliaré las oportunidados oducacionalos y combatría las discriminacionos on el acceso y permanencia on los estudios; porque, adomás, la onsoñanza quo on olla se impartirá ostará basada on las mojoros tradicionos democráticas del pueblo de Chilo.

3.5. Pluralista, porquo no scré vchículo do imrosición doctrinaria, sino que buscará hacer do la oducación una taroa libortaria on quo ol educando crezca y formo su propio modo de pensar, a travós do un trabajo podagógico croativo quo lo onfronto a la reolidad en forma crítica y ciontifica.

3.6. Productiva, ya que valorizará el trabajo socialmonte útil incorporándolo teórica y prácticamonto a la formación del educando y porque contribuírá a dostorrar la nontalidad consumidora individualista y a dosarrollar otra, productiva y solidaria.

3.7. Intcgrada a la comunidad, porque so sumará a las taras dol crocimicnto do la comunidad y dosarrollará con olla la nueva cultura y ol proceso oducativo do sus integrantos.

3.8. Científica y tecnológica, porquo rocogorá o intorprotaré adocuadamento la crecionto importancia do las ciencias naturalos y socialos y do la tocnologia, roubicándolas prioritariamonto on el curriculum, y porque utilizará la motodología y los logros ciontíficos y tónicos on el propio mejoramiento do la oducación.

3.9. Humanista, ya quo apoyéndoso on una concopción unitaria de la cultura quo incorporo la cioncia y la tocnología a los logros do la humanidad, so propondrá aportar docisivamonto a la tarea social do moldear nuovas eneneracionos do chilonos intogral y armónicamonte dosarrollados; $\mathrm{y}$ 
3.10. Planificada, porque se construirá sujotándoso a los marcos globalos do la planificación dol dosarrollo nacional, procurando aprovochar racionalmonto los rocursos que le entregue la sociedad para el cumplimionto do sus motas.

\section{$4 .-\underline{\text { OBJETIVOS }}$}

\subsection{Objetivos Goneralos.}

Con ol procoso de crcación do la Escucla Nacional Unificada, insortado on ol procoso de construcción de una sociodad socialista, democrática y humanista, so intonta cumplir con los siguiontos objotivos do la politica do domucratización oducacional on quo esté compromotido ol Gobierno Fopular.

4.1.1. Contribuir a afianzar ol naciento sistoma social do vida propiciando, por una parto, olovar la capacidad do organización y unidad dol puoblo on función do los grandos objotivos $y$ taroas dol procoso do cambio revolucionario $y$, por otra, favorocer una roal participación do las mayorías on la construcción do la nuova sociodad, haciondo do cada chilono un agonto innovidor y promotor do cambios socialas.

4.1.2. Dosarrollar la capacidad cientifica y tócnica dol puoblo quo lo porinita controlar los mocanismos do podor do la sociodad para. hacor roalidad una socicdad do participación.

4.1.3. Promovor un profundo procoso de incorporación masiva dol puoblo quo pornita asegurar ol accoso y disfruto do la cultura y la oducación por parto do las mayorias haciondo dol derocho a la oducación una roalided concrota do cada chilono.

4.1.4. Proporcionar al sistema cducacional la flexibilidad y capacidad do roadaptación áéli y dinámica, indisponsablo para rosponder a loi roquorimiontos quo constantomonto estará formulando al procoso do construcción do la nueva sociodad, lo quo oxigirá una actividad pormanonto de planificación, ovaluación y roformulación on los diforontos aspoctos dol procoso oducativo.

4.1.5. Oriontar ol procoso oducativo on los principios do unidad do tooría y prpactica y do ostudio y trabajo productivo, on tórminos quo pormitan la formación do un puoblo capacitado para suporar ol subdosarrollo y dotado do la enorgía croativa nocosaria para dar nacimionto a una cultura auténtica y propia.

4.1.6. Hacor posiblo, quo las univorsidados puodan dosarrollar la cioncia y la tócnica on los más altos nivolos do calidad y oxcoloncia, al liborarlas de las presionos y distorsiones provoniontos do la actual orientación dol sistoma oducacional que las ha limitado a coronar la oducación rogular y los ha oxigido una aceptación masiva de postulantos. Il ingroso a las univorsidados doborá fundarso on la capacidad objetivamento rodida do los postulantes, a partir do la crcación do condicionos roalos do igualdad on ol contoxto do una domocracia sucialista. 


\subsection{Objotivos espocíficos.}

4.2.1. Desdo ul punto do vista podagóeico, la Escuola Nacional Unificada tondoré a la formación armónica do la porsonalidad do los niños, adolescontos y jóvonos chilonos a fin do convortirlos on constructoros activos do la nucva sociodad. Así la Escucla Nacional Unificada garantizará a sus alumnos cl dusarro110 intoloctuel, físico, noral, ostético y tócnico por modio do la adocuada combinación on tro onsoñanza gonoral y politécnica, tendionte osté última, a la proparación do los oscolaros para una actividad laboral concrota.

4.2.2. Dosarrollar on los ostudiantos habilidados, concoptos, hábitos, opinionos, actitudos y valoros favorablos al trabajo coloctivo, a la convivoncia domocrática y al compromiso social, favorociendo, proforontomonto, ul contacto dirocto con la claso trabajadora y su roalidad socio-oconénica y laboral.

4.2.3. Propondor a la crodción do condiciones adocuadas do salud para tode la población y on ospocial do los ostudiantos a travós d.o un procoso curricular vital un quo so do propondorancia a las actividados doportivos, a las jornadas do oducación sanitaria, a la vida al airo libro, como asimismo, a la protocción do ároas vordos y contros do osparcimionto y rocroación como baso do la formación do una oquilibrada porsonalidad on la juvontud.

4.2.4. Lcontuar ol valor dol trabajo como ol monto activo un la formación do la nuova sociodad, croando on los jóvonos ol rospoto al trabajo físico, dejando do considorar a ésto como una actividad do nivol inferior.

4.2.j. Crcar una concioncia nacional, libro y soborma, con facultad para buscar on los túrminos do la rolación solidaria con los otros pucblos latinoamoricanos y ol rosto dol mundo nuovas ostructuras do convivoncia intornacional.

4.2.6. Ixaltar la nacionalidad por modio dol cultivo do los valoros y produetos culturalos autóctoncé, il mismo tiompo que so incorporon olomontos do la cultura univorsal quo pormitan onriquocor ol acorvo nacional y la participación dol puoblo on el arto, la literatura, las ciencias, la tocnología y los modios do comunicacion.

4.2.7. Dosarrollar on la juvontud una concopción ciontrfica do la sociodad, dol hombro $\mathrm{y}$ do la naturaloza, quo asceguron una ofoctiva participación on ol desarrollo social.

4.2.8. Contribuir al dosarrollo armonico do la pursonalidad do los jóvonos un los valoros dol humanismo socialista.

4.2.9. Proporcionar una oducación gonoral y politócnica quo rosponda a los roquorimiontos do la r lanificación nacional y rogional haciondo posible quo la juvontud cumpla un rol activo on la vida del trabajo.

4.2.10. Contribuir a cambiar la montalidad consumidora propia do la sociodad capitalista por un focundo ospírita do solidaridad humana.

4.2.11. Atunder las nocosidados dol proproso regional y looal on una acción planificada y dosarrollada con la comunidad, como una manora do propendor a arraigar a los jóvenos a su rospoctiva rogión. 


\section{ISTRUCTURA.-}

Corresponderá a la FNU. la atención de 1 a educnción parvularia y la educación general y politécnica.

\subsection{Educación Parvularia}

5.1.1. Los niños de 0 a 6 años estarán atendidos por las Salas Cunas y Jariines Infantiles.

Cualesquiera soa su dependoncia administrativa, estos otganismos ostarán sujetcs a la tuición técnica del Ministerio de Exucación on 10 roferente a sus actividados cducativas, a la oficiencia y ca lidal de su perscnal $y$, on general, a la conducción ciontífica del proceso de desarrollo del niño en la más inportanto fase de su exis toncia.

Las acciones que se pronueven para expandir la atención y mejorar cualitativanonte la oducación on este nivel, adquieren gran importancia dada la creciente participación do la mujer on las tareas do la producción, que la obligan a abandonar parciallonto las responsa bilidades inherentos al hogar. Es te fonómeno so acontuará más aún on los próximos años.

El contenido de la oducación parvularia deberé constituir la base fundamental dol proceso integral de formación del individuo. Deberé crearso la máxima articulación entre ssta y $1:$ educación general y politécnica.

5.1.2. La acción do la oducación parrularia se extendorá más allá do los propios niños para llegar a los parros, a la familis y a la comuni dad, medianto programas integrados y pemanontes do educacion foniliar.

\subsection{Piducacion General y Politónica.}

5.2.1. La Educación General y Politécnica creará las condicionos para que educandos, trabajadoros do la oducación y comunidad participon on la creación colectiva de los biunes culturales que necosita nuestra sociedaù. Ello implicará que los oducandos conocerán creando y no sólo consumirán cioncia sino que contribuirán a producirlağ no solo disfrutarín de los valoris del arte y $l a$ cultura sino que aportarón a su desarrollo y enriquocimionto.

La Educación Gener y y Politêcnica ayudará a la adquisición de los fundamentos cientificos do las ramas nás importantes de la producción de bionos $y$ de eerralos, al dominio teórico y práctico de la tecnologia y al conocimiento y solución do los problemas sociales que afectan al país. Esto so logrará por medio de la íntima vinculación ontro la escucla y la vida, la ensonanza y la producción, la teoría y la práctica, haciendo de este modo la educación más vital, el conocimiento mús ciontífico, el lesarrollo más pleno, y ascgurando cn cada joven la formación del hombre, del ciudadano y del productor. 
5.2.2. Atendorá a los jóvenos de 6 a $\mathrm{k} 8$ años. En principio tondrá una duración de 12 años y se expresará como un procesc continuo que atienda las necesi dades del desarrollo nacional y asimismo rosponda a las características del creciniento psicobiologico del estudiante y a sus potencialidades, a travós le una adecuada organización curricular.

5.2.3. Para facilitar la edebación del curriculun a las característioas de las diversas otapas del desarrollo del alumo y permitir una mojor planifica ción de la ElvU, este ciclo se estructurará on 4 tramos:

5.2.3.1. De $1^{\circ}$ a $4^{\circ}$ año, cuyos contenidos curriculares se organizarán un forma integrade (unidades);

5.2.3.2. De $5^{\circ}$ a 60 año, en el cual se cerminara el sistema globalizado con la organización de contenidos por ́reas y disciplinas;

5.2.3.3. De $7^{\circ}$ y $3^{\circ}$ año, cuyo curriculum gradualmente armonizars la or nización de contenidos por áreas con la de disciplinas y enfati zaré ol proceso do orientación; y

5.2.3.4. De $9^{\circ}$ a $12^{\circ}$ año, con un curriculum estructuralo a base de 3 pla nes, común, electivo y de espocialización, intensificando gradualinento la formación tecnológica.

De ninguna manere podrá entenderse que esta forma le organización do los contenidos da lugar al establocimion o do nivolos quo rompun el caráctor unitario y continuo de la educación gonural y politécnica.

Las modalidados de organización ihdicadas estarín prosentes a 10 Isrgo de todo el proceso escolar. Th cada unc de los tramos, una o dos de ellas tendrán especial relevancia por sobre las denás, un consiaración a las caractoristicas que va presontando el desarncllo psicobiológico y social del educando.

La división on cursos anuales tampoco podrá entendersc cono dofinitiva. El caráctor do ensayo de la ENU facilitará la búsqueda do ctras formas dc organiración curricular on cl tiempo, talos como somestros, grupos de años, avance por niveles, ctc.

5.2.4 Los siguientes son al gunos rasgos especificos del curriculum do la educación general it politécnica.

5.2.4.1. El curriculum y la organización y adninistración de la ENU debe rín consultar on sus primoros ocho años modalidades durante las cuales los alumnos podrán libremonte elogir sus actividados, tanto pare favcrecer el desarrollo de aptitudes, habilidados e intereses especiales, como para facilitar recuperaciones. 
5.2.1.2. La formación gencra.l y J as oxporiernoias obtenidas en el proceso estudio-trabajo, en las prácticas productivas, en los trabajos voluntarios en la comunidad facilitarán al alumno la continuación de sus estudios o su incorporación a la vida familiar, democrática y productiva de la comunidad.

5.2.4.3 La formación tecnológica no solo se adquirirá en los laboratorios y talleres de los establecimientos escolares sino también en las industrias y servicios de la comunidad, como par te integral de la docencia..

5.2.4.4 Desde el $I^{\text {er }}$ año se dará énfasis a las actividades de orienta cion para favorecer el conocimiento de todas las áreas tecnolé gicas a través del proceso de estudio-trabajo y de los trabajos voluntarios, lo que dará oportunidad para observar y registrar las experiencias $y$ preferencias de cada estudiante.

5.2.4.5 El proceso de orientación culminara en el $9^{\circ}$ año, debiendo en es te curso darse a los alumnos la oportunidad de conocer mejor las diversas familias de especialidades que ofrece el cam po educacional, mediante una orientación jeneral técnica en cada una de ellas $y$ con la participación directa en sus acti vidades. Por medio de un sistema rotativo los grupos de alumnos de $9^{\circ}$ deverán cubrir el proceso antes indicado en cada una do las familias o áreas de especialidades, además de observacio nes, prácticas obligatorias y trabajos voluntarios.

5.2.4.6. Los propositos de los 3 plenes de los cursos $9^{\circ}$ a $13^{\circ}$ son $10 s$ siguientes:

a. El plan común tiene por objeto asegurar una base cultural mínima tanto para la continuación de estudios como para la in corporación a la vida familiar y de la comunidad.

b. El plan electivo está destinado a permitir que los alumnos tengan la posibilidad de elcgir contenidos curricularos acordes con sus aptitudes, intereses y habilidades.

c. El plan de especialización comprenderá las asiunaturas ligadas a la especialidad (profesionales) olegida y las actí vidades prácticas de las mismas.

5.2.4.7. En el $10^{\circ}$ año cada alumno reducirá su actividad tecnológica a una sola área además del plan comín y del electivo. Asi intensificará su conocimiento teórico sobre el área y acentuará las prácticas supervisadas en las difersas especialidades que ella cubre.

5.2.4.8. En los $11^{\circ}$ y $12^{\circ}$ adomás del plan común y del electivo, el alum no se concontrará en lo tecnológico en una sola especialidad.

5.2.4.9. Eval aciones y estudios posteriores permitirán decidir si el comienzo de la especialidad so adelanta o se posterga y si se sará neceseria la creación de un $13^{\bullet}$ año pare las especialida des de mayor complojidad.

5.2.4.10 En el proceso constante de evaluación que deberá realizarso des do la caucación parvularia se dejará constancia de los rendimientos, prácticas productivas, trabajos voluntaricis, además de apreciaciones generales sobre aptitudes, habilidades, acti tudes e intereses de los alumnos. 
5.2.4.11. Para los alumnos que no continúan sus estudios en el área de educación regular habré cursos especiales de superación cultural y orientación laboral que les permitan obtener una calificación.

5.2.4.12. Los alumnos que por cualquier razón o motivo deban abandonar la escuela podrán reincorporarse al nivel que corresponda de acue rdo al desarrollo logrado fuera de ella.

5.2.4.13. Al tórmino dol proceso, los estudiantes recibirán el título do Sub-teohtoo oude Técnico de Nivel Medio segrín corresponda y un certificado que acredite sus rendimientos, trabajos voluntarios y una apreciacion general sobre los diversos aspoc tos de su personalidad. 